\title{
Wavefront sensing and control performance modeling of the Thirty Meter telescope for systematic trade analyses
}

Carl Nissly, Byoung-Joon Seo, Mitchell Troy, Gary Chanan, Scott Roberts, et al.

Carl Nissly, Byoung-Joon Seo, Mitchell Troy, Gary Chanan, Scott Roberts, John Rogers, "Wavefront sensing and control performance modeling of the Thirty Meter telescope for systematic trade analyses," Proc. SPIE 9150, Modeling, Systems Engineering, and Project Management for Astronomy VI, 91500S (4 August 2014); doi: 10.1117/12.2057157

Event: SPIE Astronomical Telescopes + Instrumentation, 2014, Montréal, Quebec, Canada 


\title{
Wavefront Sensing and Control Performance Modeling of the Thirty Meter Telescope for Systematic Trade Analyses
}

\author{
Carl Nissly ${ }^{a, b}$, Byoung-Joon Seo ${ }^{a, b}$, Mitchell Troy ${ }^{a, b}$, \\ Gary Chanan ${ }^{c}$, Scott Roberts ${ }^{d}$, John Rogers ${ }^{d}$ \\ ${ }^{a}$ Jet Propulsion Laboratory, Pasadena, CA 91109, USA \\ ${ }^{b}$ California Institute of Technology, Pasadena, CA 91125, USA \\ ${ }^{c}$ University of California, Irvine, CA 92697, USA \\ ${ }^{d}$ Thirty Meter Telescope Observatory, Pasadena, CA 91107, USA
}

\begin{abstract}
We have developed an integrated optical model of the semi-static performance of the Thirty Meter Telescope. The model includes surface and rigid body errors of all telescope optics as well as a model of the Alignment and Phasing System Shack-Hartmann wavefront sensors and control algorithms. This integrated model allows for simulation of the correction of the telescope wavefront, including optical errors on the secondary and tertiary mirrors, using the primary mirror segment active degrees of freedom. This model provides the estimate of the predicted telescope performance for system engineering and error budget development. In this paper we present updated performance values for the TMT static optical errors in terms of Normalized Point Source Sensitivity and RMS wavefront error after Adaptive Optics correction. As an example of a system level trade, we present the results from an analysis optimizing the number of Shack-Hartmann lenslets per segment. We trade the number of lenslet rings over each primary mirror segment against the telescope performance metrics of PSSN and RMS wavefront error.
\end{abstract}

Keywords: Wavefront Sensing and Control, Segmented Mirrors, Optical Modeling, MACOS, Normalized Point Source Sensitivity, Thirty Meter Telescope, Alignment and Phasing System, Shack-Hartmann Wavefront Sensing

\section{INTRODUCTION}

We have updated our optical model of the Thirty Meter Telescope (TMT) using the Modeling and Analysis for Controlled Optical Systems (MACOS) software ${ }^{1}$ for system engineering performance and sensitivity analyses for static optical errors. ${ }^{2}$ The TMT design is a highly segmented mirror telescope with an equivalent aperture of 30 meters. The F/1 primary mirror is made up of 492 hexagonal segments, each with a $1.44 \mathrm{~m}$ corner-to-corner width. The MACOS optical model is a high-resolution optical model of the TMT with the capability to misalign all optics in six degrees of freedom and to apply surface figure errors to each mirror. This TMT MACOS model provides a means to rapidly calculate the wavefront quality of the telescope and has been used to simulate mirror segment surface figure control, alignment and phasing methods, wavefront maintenance strategies, the M1 Control System, and to validate the optical performance error budget.

The TMT optical system errors are modeled using Matlab to calculate surface shape change data and six degree of freedom misalignment of each optic. These errors include effects related to gravity and temperature dependent telescope errors in addition to mirror polishing and temporal alignment errors. We pass these parameters to MACOS for ray-tracing and Optical Path Difference (OPD) calculation at the exit pupil. The Matlab suite of functions for calculating these system perturbations is divided into three main parts, one for the M1 segments, M2, and M3. Each of these functions takes the inputs of telescope angle, delta temperature, and a series of options for running parametric studies and isolating system effects. The outputs of these functions are then the parameter vectors in the MACOS preferred format ready for the OPD calculation. After MACOS calculates the wavefront at the TMT exit pupil, the map is passed to a Matlab postprocessor function. This routine

Further author information: (Send correspondence to Carl Nissly, Carl.R.Nissly@jpl.nasa.gov)

\footnotetext{
Modeling, Systems Engineering, and Project Management for Astronomy VI, edited by George Z. Angeli, Philippe Dierickx, Proc. of SPIE Vol. 9150, 91500S

(C) 2014 SPIE $\cdot$ CCC code: $0277-786 X / 14 / \$ 18 \cdot$ doi: $10.1117 / 12.2057157$
}

Proc. of SPIE Vol. 9150 91500S-1 
then calculates the Point Spread Function (PSF) and image metrics such as the RMS wavefront error (rmsWFE) both before and after Adaptive Optics (AO) correction, Normalized Point Source Sensitivity (PSSN), plate scale distortion, pupil shift, and pointing error. The PSSN is a multiplicative image performance metric that quantifies the science loss for seeing limited instruments ${ }^{3}$ in terms of required increase in integration time. As an example, a PSSN of 0.85 means that, to reach the same sensitivity levels, a $17.6 \%$ longer integration time will be required relative to a perfect system. (The integration time scales by 1/PSSN.)

We have recently added the capability to the TMT MACOS model to include the Alignment and Phasing System (APS) ${ }^{4,5}$ Shack Hartmann Wavefront Sensor (SH-WFS) and control algorithms. This provides the ability to simulate the post calibration performance for optical errors on the secondary and tertiary mirrors after correction by the active degrees of freedom of the primary mirror segments and secondary mirror. The primary mirror segments have 21 Warping Harness (WH) actuators per segment that can deform the segment surface as well as three degrees of rigid body motion (piston, tip, and tilt). The secondary mirror can be moved in all 6 degrees of rigid body motion.

In this paper we will describe updated performance estimates which include this APS calibration model of error correction using Wavefront Sensing \& Control (WFSC). Section 2 defines the wavefront sensor models and control simulation framework. Section 3 describes the updated static optical error performance after APS calibration in terms of PSSN. Section 4 includes a trade study of the SH-WFS design using WFSC corrected performance as a metric.

\section{WAVEFRONT SENSING AND CONTROL SIMULATION}

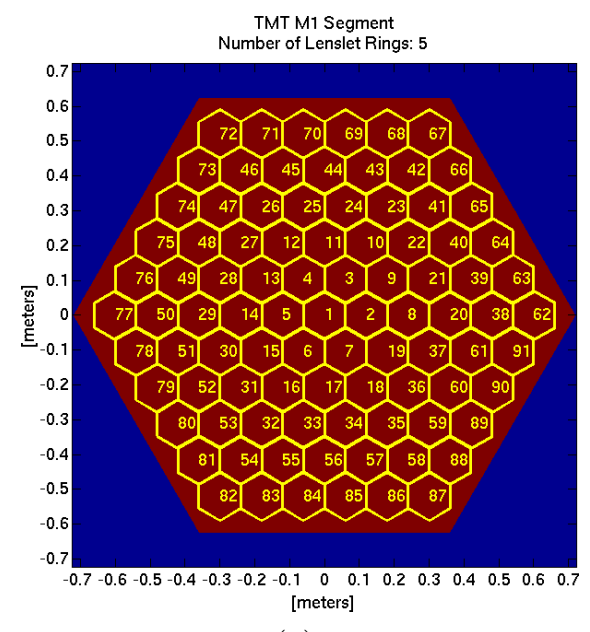

(a)

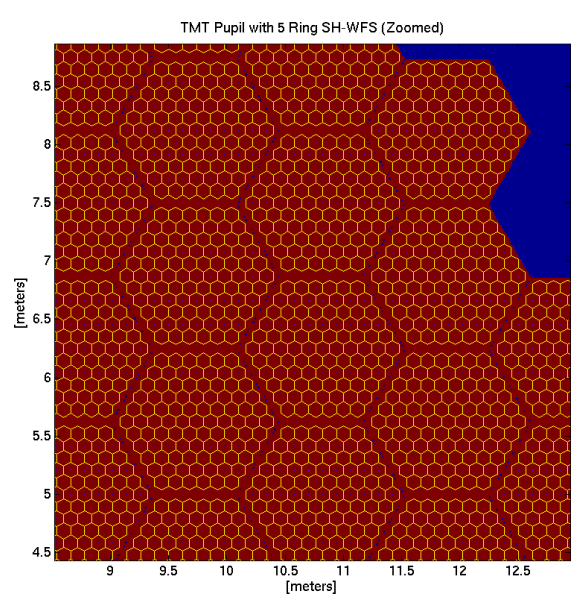

(b)

Figure 1: 5 ring SH-WFS geometry

\subsection{Wavefront Sensing}

We have implemented both a SH-WFS and an "Ideal-WFS" for error budgeting verification purposes. The "Ideal WFS" is used for evaluating individual error terms while the SH-WFS is used to calculate the wavefront sensing errors related to wavefront sampling, atmospheric averaging, and calibration of the entire TMT system using the APS instrument.

The "Ideal WFS" effectively uses the simulated OPD directly for sensing the wavefront shape. This method evaluates the theoretical limit of the wavefront control correction of each input error using the telescope control degrees of freedom. Thus, we exclude any measurement errors due to non-ideal features of the wavefront sensor design.

The SH-WFS model is used to quantify the wavefront sensing errors and simulates the baseline APS instrument design. This 5-ring SH-WFS configuration has 91 hexagonal sub-apertures per segment, with $28 \mathrm{~cm}$ 
sub-apertures from corner-to-corner in entrance pupil space. Over the $492 \mathrm{M} 1$ segments, this works out to be 44,772 Shack Hartmann ( $\mathrm{SH}$ ) subimages across the pupil. Figure 1 shows the lenslet geometry across a single segment and a zoomed in view of the lenslet grid sampled across the TMT pupil. Our model samples the wavefront as seen by each sub-aperture, fitting tip and tilt to each sample to measure slope. The dominant noise source from the SH-WFS measurement is residual atmospheric error. To simulate this effect, a long exposure phase screen ${ }^{5,6}$ is added to the calculated telescope wavefront prior to the SH-WFS slope measurement. An effective integration time of 300 seconds is assumed for the measurement error. This model includes the effects of atmospheric noise, spatial sampling and aliasing of the SH-WFS. The model does not include relatively minor effects such as centroiding errors or CCD sampling.

\subsection{Wavefront Control}

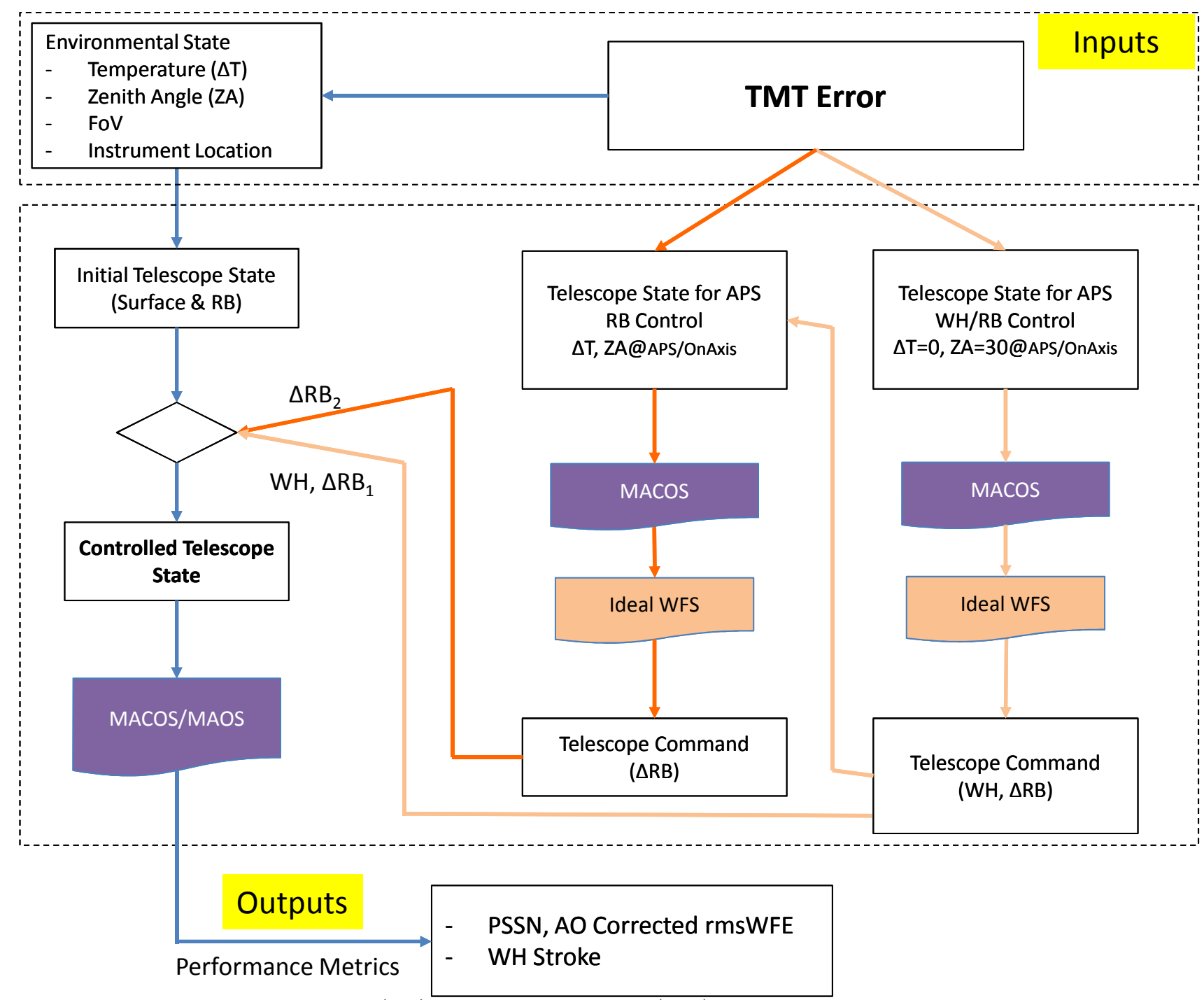

Figure 2: Rigid body (RB) and Warping Harness (WH) look-up-table calibration procedure.

The Wavefront Control (WFC) portion of the simulation is designed to replicate the TMT calibration procedure using APS. Figure 2 shows the overall simulation process used. The right-hand column of Figure 2 shows the initial part of this calibration procedure. Here the optimal segment $\mathrm{WH}$ actuator positions and the M1 \& M2 segment Piston, Tip, and Tilt (PTT) positions are computed to minimize the Wavefront Error (WFE) when TMT is configured to point at the APS instrument position, on-axis, and at a telescope zenith angle of 30 
degrees. These $\mathrm{WH}$ and rigid body actuator commands are stored at the time of calibration and applied to each computed telescope wavefront. This simulates the act of calibrating the WH at a single telescope zenith angle and temperature, which is the TMT baseline procedure.

Next, a look up table is calculated by optimizing the rigid body alignment of M2 and each M1 segment as a function of gravity and temperature. This process is shown in the center column of Figure 2. Here the segment and M2 PTT positions are computed to minimize the WFE as measured over a range of telescope zenith angles and delta temperatures from the time of initial APS calibration. These rigid-body commands are stored at the time of calibration and later applied to each computed wavefront with the same zenith angle and delta temperature, independent of instrument position.

We now have calculated a set of APS calibration tables that are applied when simulating TMT operation at all input configuration conditions. For any input zenith angle, delta temperature, instrument, and field angle we then use MACOS to calculate the wavefront including the control commands computed from the APS calibration tables. (For example, the rigid body and segment WH commands calibrated at the APS instrument for the same zenith and delta temperature are applied when the telescope is pointed at the NFIRAOS or MOBIE instrument positions.) This corresponds to the left-most column in Figure 2.

Figure 3 shows the WFSC algorithm that uses the wavefront sensors and control methods described above. This WFSC loop is set up to correct the WFE based upon each telescope OPD calculated by MACOS. The output of a simple integrating controller and sensitivity model calculates the commands necessary to correct the wavefront. These new states are passed back to MACOS to determine a new telescope OPD. After the convergence criteria have been met, the resulting control commands are returned to the overall simulation described in Figure 2. When calculating the WFC commands, the degrees-of-freedom applied are M2 PTT, segment PTT, and segment WH actuators (10 WH Singular Value Decomposition (SVD) modes are controlled using a combination of the $21 \mathrm{WH}$ actuators on each segment). For the "Ideal WFS", the OPD itself and therefore the rmsWFE is minimized using the control degrees of freedom. For the SH-WFS, the SH slope is used as the metric for WFC.

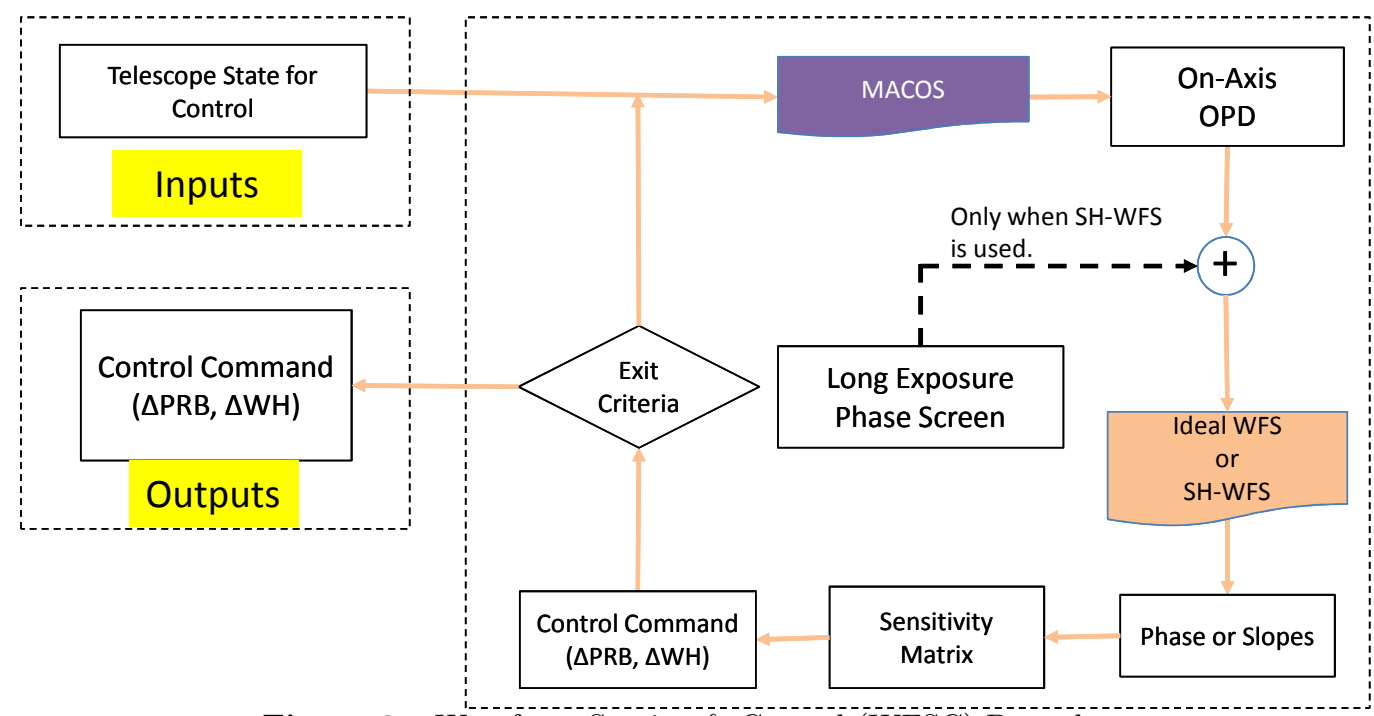

Figure 3: Wavefront Sensing \& Control (WF SC) Procedure

\section{STATIC OPTICAL AND WAVEFRONT SENSING ERROR PERFORMANCE}

By adding the capability to sense and control the wavefront error using the M1 and M2 control degrees of freedom, we then have the opportunity to update the current best performance estimates for the modeled terms. Our previous publication ${ }^{2}$ reported uncontrolled performance values for the current model at the time. Since this publication, physical models of the various effects have been updated so a direct comparison is not available. However, adding M1 \& M2 control of the error terms improves the performance estimates. We have 
also included the closed loop AO performance estimates, which further improves the wavefront using the TMT AO Narrow-Field Infrared Adaptive Optics System (NFIRAOS) instrument. The principal wavefront correction by NFIRAOS is achieved via a 60x60 actuator Deformable Mirror (DM) in the first generation instrument and a planned 120x120 actuator DM in a future instrument update.

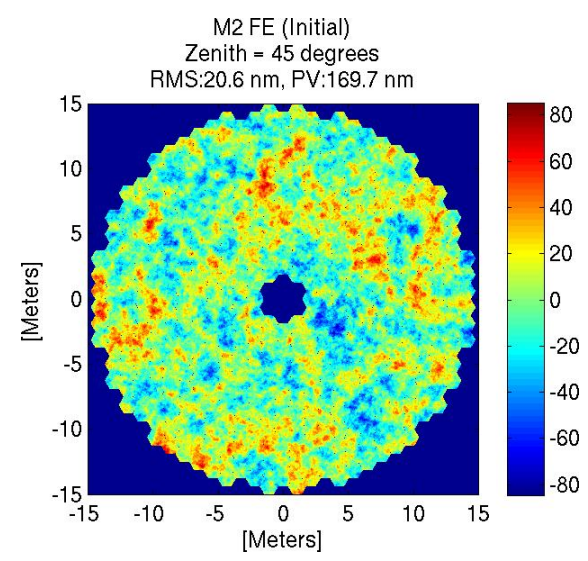

(a)

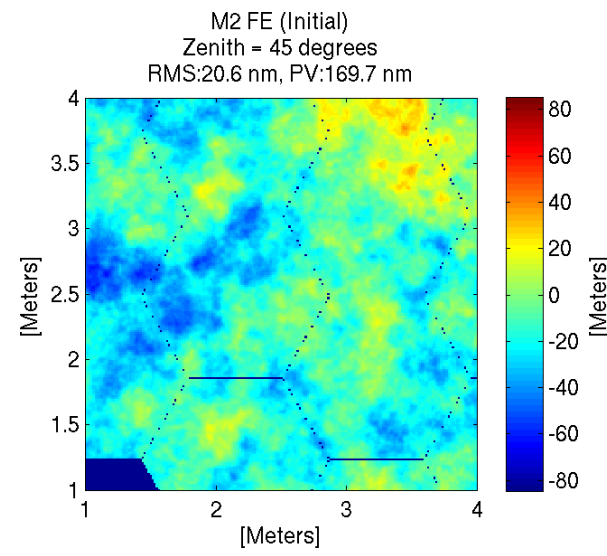

(d)

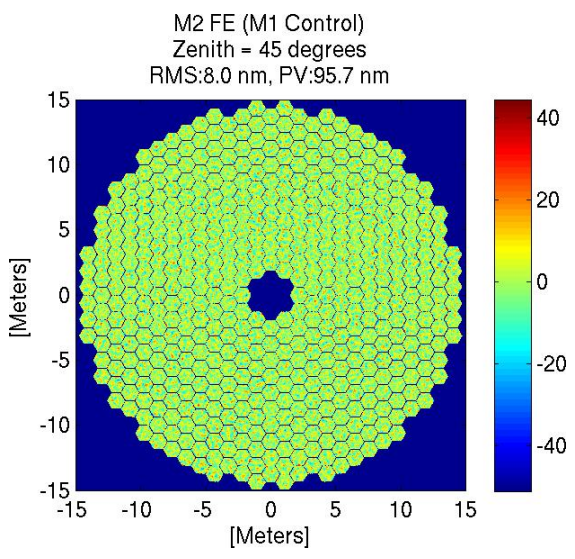

(b)

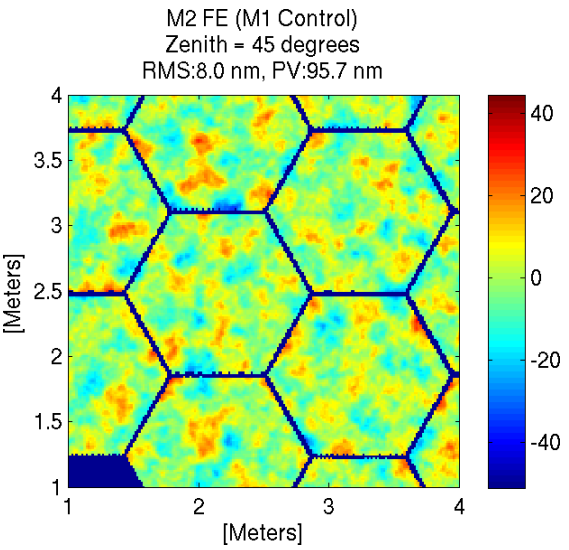

(e)

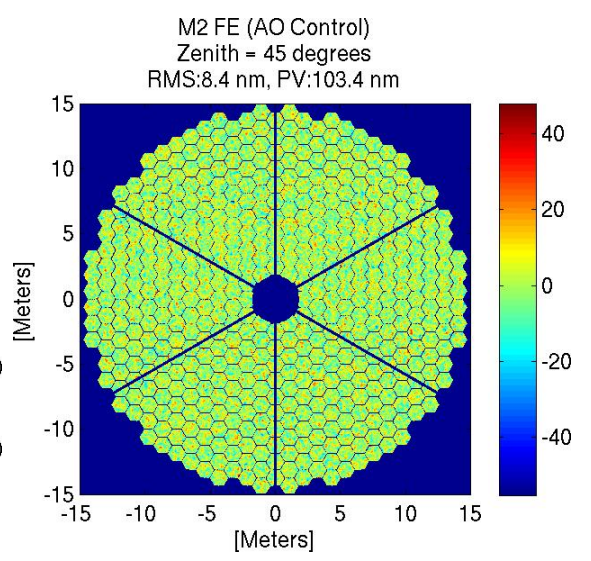

(c)

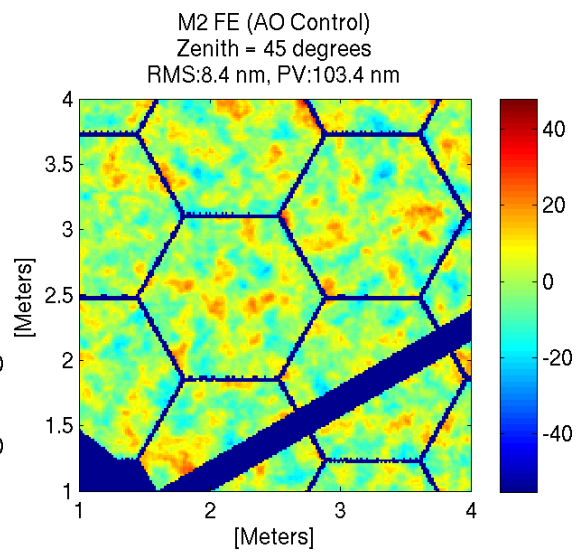

(f)

Figure 4: M2 Residual Figuring Error (M2RFE) : OPD wavefront maps

As an example, Figure 4 shows the wavefront before and after WFC for the M2RFE. This is the current model of the residual polishing error of the TMT secondary mirror. The polishing error is modeled using the current TMT polishing specification which is based on a SlopeRMS measurement. ${ }^{7}$ To model the residual polishing error, we numerically generate a von-Karman Power Spectral Density based surface and rescale the amplitude to just meet the SlopeRMS measurement. Figure 4 shows the correction of the wavefront from $20.6 \mathrm{~nm}$ to $8.0 \mathrm{~nm}$ using the M2 and M1 control degrees of freedom. The reduction of the mid-spatial frequencies by the control process is clearly observed. Figure 4 also shows the AO correction of the M1 controlled wavefront. As expected, the residual polishing error after M1 control has high spatial frequency content that is not improved by the 60 actuator AO system. Some aliasing occurs, which slightly raises the rmsWFE from 8.0nm to 8.4nm. Figure 5 shows the PSSN after M1 control to be 0.9906 at zenith pointing and an improvement in the rmsWFE AO correction from $8.5 \mathrm{~nm}$ to $7.1 \mathrm{~nm}$ by increasing the order of the DM to 120 actuators.

Table 1 presents the updated results for static optical and Wavefront Sensor (WFS) related errors in terms of PSSN and AO corrected rmsWFE for both instrument designs. The errors are broken up into mirror groups with performance estimates for both individual and combined errors. The combined error estimates are reported using the multiplication of individual PSSN values or Root Sum Square (RSS) of individual AO rmsWFE. This is done to avoid the effects induced by possible modal cancellation when combining random error terms. A separate 


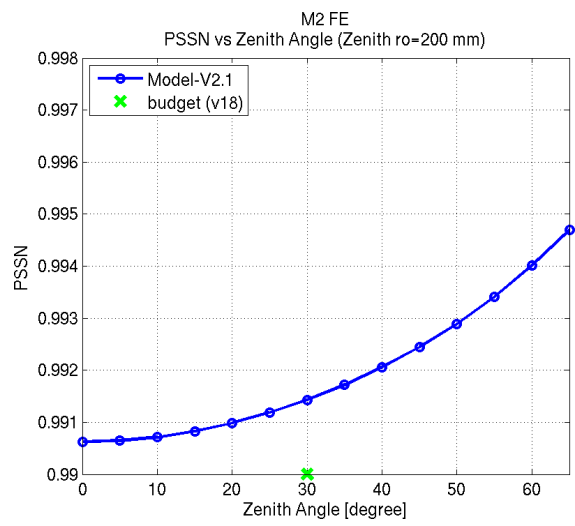

(a)

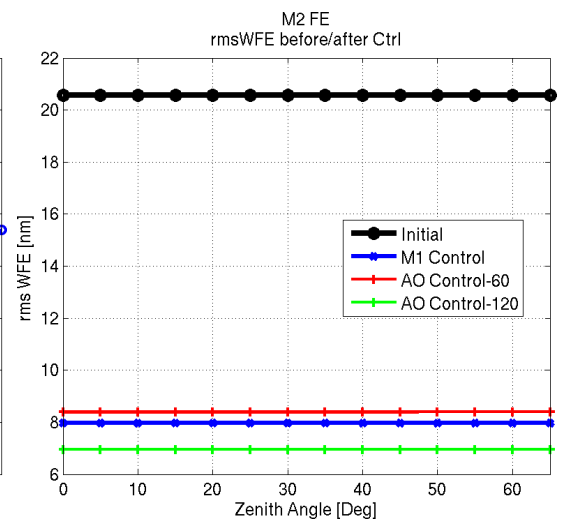

(b)

Figure 5: M2 Residual Figuring Error (M2RFE) performance. (PSSN increases with telescope zenith angle as effective $r_{0}$ decreases.)

Monte Carlo study of random seeding is currently underway to determine the proper values to represent the average of the combined errors. The individual terms that are included in the model are the semi-static optical error terms that are estimated to have the largest science impact in the seeing limited TMT error budget. The simulation conditions for the values included in the table are at a zenith angle of 60 degrees, $\Delta \mathrm{T}$ of 4 degrees from calibration temperature, $\lambda=500 \mathrm{~nm}$, and an $r_{0}$ of $200 \mathrm{~mm}$ at the zenith. The zenith angle of 60 degrees is used to demonstrate the relative performance of each error term since many error terms are calibrated out at zenith $=30$ degrees. Seeing limited PSSN performance values are reported at the APS instrument position to compute performance of the WFSC scheme described in Section 2. As M3 rotates to the NFIRAOS instrument, which is 5.5 degrees off of the elevation axis, the correction degrades as the beam footprint rotates. The AO corrected rmsWFE performance is reported at the NFIRAOS instrument position and captures this beam rotation effect.

The performance estimates in Table 1 show the relative impact on TMT performance for each of the modeled error terms. The combined PSSN for M1, M2, and M3 are 0.9321, 0.9932, and 0.9991 respectively. This indicates that the M1 static optical errors are responsible for the largest PSSN degradation of 0.9321 or a $6.79 \%$ loss in seeing limited science. The biggest two individual terms that contribute to the M1 error degradation are the Segment Residual Figure Error and Segment Support Print Through. These are high spatial frequency errors across each M1 segment that are not well-corrected by the M2 and M1 control or the AO control. The M2RFE, shown in Figure 4, currently has the largest impact on performance from the individual M2 error terms. The M3 errors are well-corrected by M1 and M2 control at the on-axis APS instrument. These errors are different from the others in that they shift as the position and orientation of the beam footprint moves across the elliptical M3 for different instruments and off-axis field angles. These effects have been taken into account when specifying the M3 polishing error, which is limited by the AO rmsWFE performance rather than the PSSN. The AO corrected rmsWFE for the combined M1, M2, and M3 errors are $27.5 \mathrm{~nm}, 8.9 \mathrm{~nm}$, and $4.2 \mathrm{~nm}$ respectively for the 60 actuator system. These improve for the 120 actuator DM system to $24.1 \mathrm{~nm}, 7.2 \mathrm{~nm}$, and $2.8 \mathrm{~nm}$ for M1, M2, and M3 combined errors. The largest error sources for the closed loop AO performance are due to the same terms that contribute to the largest reductions in the PSSN terms. The overall AO performance improvement for the M123 combined static optical errors between the 60 and 120 actuator systems decreases from $29.2 \mathrm{~nm}$ to $25.5 \mathrm{~nm}$ or an improvement of an additional $14.2 \mathrm{~nm}$ in quadrature.

The performance for the M1, M2, and M3 error terms are reported using the "Ideal WFS" to avoid including WFS errors within each term. The WFS error terms account for the inaccuracy in determining the correct segment tip and tilt positions and segment warping harness shape correction settings due to integrating over the atmosphere through the SH-WFS lenslets. (An integration time of 300 seconds is used for atmospheric averaging.) To simulate these effects, we first calculate the sensing correction of the combined static telescope errors described in Section 2.1 using the "Ideal WFS". Next, we calculate the wavefront sensing and correction procedure for the same input errors, but use the SH lenslets to measure the wavefront while also applying a 
time averaged atmospheric phase screen to each measurement. After completing both of these sensing and correction processes for the same input error, we then have a set of segment rigid body actuator and segment warping harness commands for both the "Ideal WFS" and the SH-WFS. The wavefront difference that these control commands induce provides estimates of the WFS error terms. The TMT error budget separates the overall WFS error into the effects of M1 Segment Phasing Wavefront Measurement Error, Low Order Wavefront Measurement Error (WFSLO), M1 Segment Tip/Tilt Measurement Error (WFSTT), and M1 Warping Harness Wavefront Measurement Error (WFSWH). WFSLO is defined as the low order wavefront sensing errors in estimating global Zernikes 4-15 across the TMT pupil. The two most significant impacts on PSSN and AO corrected rmsWFE are due to the WFSWH $(0.9948,10.6 \mathrm{~nm}, 6.0 \mathrm{~nm})$ and WFSTT $(0.9955,8.1 \mathrm{~nm}, 5.5 \mathrm{~nm})$. These are the errors due to the inaccuracy in determining the correct segment warping harness shape correction settings (WFSWH) and segment tip and tilt positions (WFSTT) due to the atmosphere through the SH-WFS.

Table 1: TMT Performance Simulation Results: zenith angle $=60$ degrees, $\Delta=4$ degrees, $\lambda=500 \mathrm{~nm}$, zenith $r_{0}=200 \mathrm{~mm}$, Normalized PSS results measured at APS instrument, AO corrected rmsWFE measured at NFIRAOS instrument.

\begin{tabular}{|c|c|c|c|c|}
\hline & Optical Error & $\begin{array}{l}\text { Normalized } \\
\quad \text { PSS } \\
\text { (APS Instr) }\end{array}$ & $\begin{array}{c}\text { AO Corrected } \\
\text { rmsWFE 60x60 } \\
\text { Act DM } \\
\text { (NFIRAOS Instr) }\end{array}$ & $\begin{array}{c}\text { AO Corrected } \\
\text { rmsWFE 120x120 } \\
\text { Act DM } \\
\text { (NFIRAOS Instr) }\end{array}$ \\
\hline \multirow[t]{6}{*}{ M1 } & $\begin{array}{r}\text { Combined Passive Support } \\
\text { Gravity Segment }\end{array}$ & 0.9605 & 22.5 & 20.7 \\
\hline & $\begin{array}{l}\text { Clocking and Decenter } \\
\text { Segment Figuring Error with }\end{array}$ & 0.9994 & 3.4 & 2.0 \\
\hline & Warping Harness Correction & 0.9747 & 13.6 & 11.7 \\
\hline & Thermal Segment Distortion & 0.9964 & 7.0 & 3.6 \\
\hline & Thermal Segment Clocking & 0.9999 & 1.3 & 0.8 \\
\hline & Combined M1 Errors & $0.9321^{*}$ & $27.5 \dagger$ & $24.1 \dagger$ \\
\hline \multirow{3}{*}{ M2 } & Combined Passive Support & 0.9996 & 2.7 & 1.6 \\
\hline & M2 Figuring Error & 0.9937 & 8.5 & 7.1 \\
\hline & Combined M2 Errors & $0.9932^{*}$ & $8.9 \dagger$ & $7.2 \dagger$ \\
\hline \multirow{3}{*}{ M3 } & Combined Passive Support & 1.0000 & 0.6 & 0.4 \\
\hline & M3 Figuring Error & 0.9991 & 4.2 & 2.8 \\
\hline & Combined M3 Errors & $0.9991^{*}$ & $4.2 \dagger$ & $2.8 \dagger$ \\
\hline M123 & Combined M1, M2, M3 Errors & $0.9250^{*}$ & $29.2 \dagger$ & $25.5 \dagger$ \\
\hline \multirow{5}{*}{ WFS } & $\begin{array}{r}\text { M1 Segment Phasing } \\
\text { Wavefront Measurement Error } \\
\text { Low Order }\end{array}$ & 0.9985 & 5.3 & 3.6 \\
\hline & $\begin{array}{r}\text { Wavefront Measurement Error } \\
\text { M1 Segment Tip/Tilt }\end{array}$ & 0.9997 & 0.8 & 0.4 \\
\hline & Measurement Error & 0.9955 & 8.1 & 5.5 \\
\hline & $\begin{array}{l}\text { M1 Warping Harness } \\
\text { Wavefront Measurement Error }\end{array}$ & 0.9948 & 10.6 & 6.0 \\
\hline & Combined WFS Errors & $0.9885^{*}$ & $14.4 \dagger$ & $8.9 \dagger$ \\
\hline M123 \& WFS & Combined Telescope Errors & $0.9144^{*}$ & $\overline{32.5 \dagger}$ & $2 \mathbf{2 6 . 9 \dagger}$ \\
\hline
\end{tabular}

*Calculated by multiplication of individual mirror error cases.

$\dagger$ Calculated by RSS combination of individual mirror error cases.

\section{WAVEFRONT SENSOR TRADE}

Since the inputs to the optical model are so extensively parameterized, it becomes a simple exercise to vary parameters and then measure their effects on telescope performance. Here we vary the number of SH rings sampled across each M1 segment to find an optimum SH-WFS sampling as an input to the APS WFS design. The APS team has developed a set of SH-WFS designs for a varying number of lenslet rings per segment. We reference these designs for this trade study and compare their performance to the "Ideal WFS" described in 
Section 2.1. Finding the proper number of lenslets per segment will balance the spatial sampling of the expected TMT errors and segment surface correctability against the complexity that comes with additional SH subimages per segment and the highest spatial frequency that is required for observation.

To evaluate the SH-WFS designs, we used the M123 Combined static telescope errors case. This OPD error is made up of the combination of all of the error terms described in Section 3 before WFC correction. Figure 6 shows the wavefront that is used at a telescope zenith angle of 30 degrees and a change in temperature of 2 degrees from the time of calibration. Before WFC correction the wavefront is dominated by polishing residual low spatial frequency errors on each segment with a total rmsWFE of $224 \mathrm{~nm}$. These astigmatic errors are highly correctable using the segment warping harnesses, which will be observed in the post correction rmsWFE.

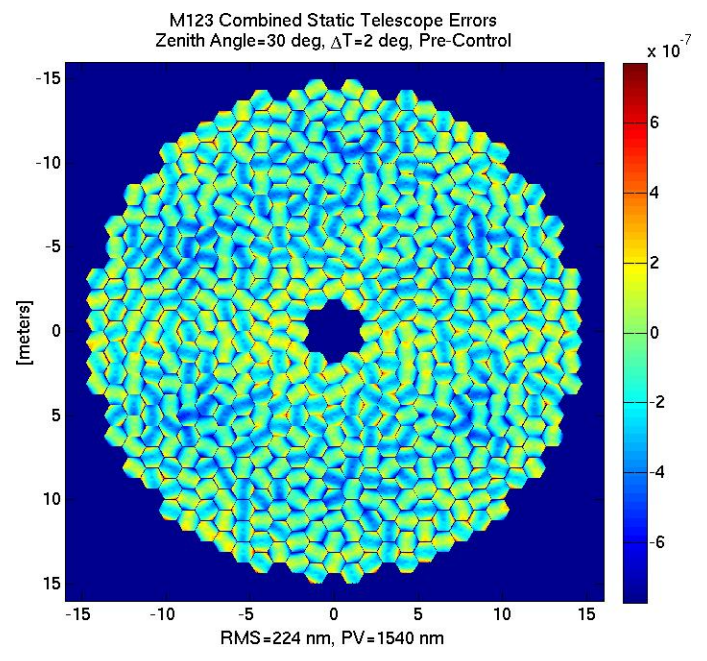

(a)

Figure 6: M123 combined static telescope error OPD

Once the input telescope wavefront is computed, we then pass the wavefront through the WFSC process described in Section 2.2 for the set of APS SH-WFS designs. We run the simulation both with and without atmospheric noise to observe the system performance in idealized and real world environments. The results of these calculations are summarized in Figure 7 and Table 2.

Table 2: WFS Performance Trade Results

\begin{tabular}{|c|c|c|c|c|}
\hline WFS Design & $\begin{array}{c}\text { PSSN without } \\
\text { Noise }\end{array}$ & $\begin{array}{c}\text { PSSN with } \\
\text { Atmosphere Noise }\end{array}$ & $\begin{array}{c}\text { RMS WFE } \\
\text { without Noise } \\
{[\mathbf{n m}]}\end{array}$ & $\begin{array}{c}\text { RMS WFE with } \\
\text { Atmosphere Noise } \\
{[\mathbf{n m}]}\end{array}$ \\
\hline 1 Ring & 0.3528 & 0.3543 & 259.9 & 263.5 \\
\hline 2 Rings & 0.9420 & 0.9373 & 21.4 & 23.1 \\
\hline 3 Rings & 0.9440 & 0.9396 & 20.7 & 22.2 \\
\hline 4 Rings & 0.9452 & 0.9409 & 21.0 & 22.4 \\
\hline 5 Rings & 0.9459 & 0.9415 & 21.0 & 22.4 \\
\hline Ideal WFS & 0.9443 & - & 19.3 & - \\
\hline
\end{tabular}

Without atmospheric noise, the "Ideal WFS" corrects the input error to a rmsWFE of 19.3nm with a PSSN of 0.9443. This shows the best performance in terms of rmsWFE but a mid range performance in terms of PSSN as compared to the SH results. This is due to the "Ideal WFS" optimization of the wavefront error itself instead of the measured slopes by the SH-WFS. The best performance in terms of PSSN is from the 5-ring SH-WFS design with a PSSN of 0.9459 and rmsWFE equal to 21.0nm before AO control. PSSN degrades slightly as the number of lenslet rings, and therefore spatial frequency range, decreases. The difference between the 5-ring and 2-ring design in terms of PSSN is 0.0039 or $0.39 \%$ normalized overall science loss to the TMT performance. The rmsWFE performance before AO control is largely the same for all SH-WFS results ranging from 20.7-21.4nm. 


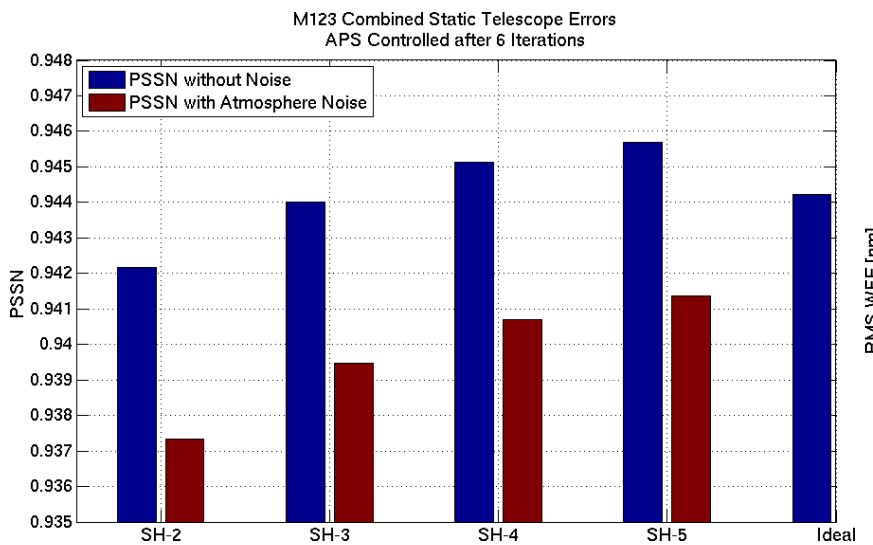

(a)

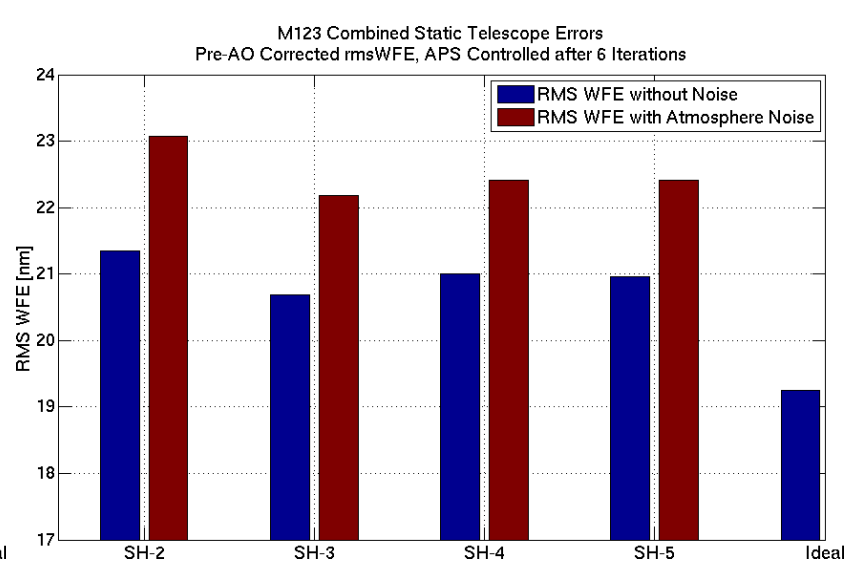

(b)

Figure 7: WFS Performance Trade Results

The relative performance of these WFS results compares well to a study of WFSC correction of single segment figuring error published by our group previously. ${ }^{8}$ The "Ideal WFS" optimizes the rmsWFE while the SH-WFS minimizes the slope measurements. This effect conveniently optimizes the PSSN and reduces the required warping harness actuator stroke.

Adding atmosphere noise to the SH-WFS design cases has a largely uniform impact across WFS designs in terms of both PSSN and rmsWFE before AO control. The PSSN reduces by a factor of about 0.9952 and the rmsWFE increases by approximately 8.0nm for each of the WFS designs. The 5-ring design's PSSN remains the best at 0.9415 and an rmsWFE of $22.4 \mathrm{~nm}$.

Table 2 also shows results for the simulation with a 1-ring SH-WFS. As expected, the sensing and control scheme breaks down for the 1-ring design providing little correction of the input errors. This is likely due to a 1-ring array not having enough measurements across the segment to sense and correct for the first 10 SVD modes as is the case with the higher order SH-WFS designs. It may be possible to tune the control algorithm to only correct for the $2^{\text {nd }}$ order SVD modes to achieve better performance. However, this is unlikely to raise the performance to be anywhere close to the TMT performance requirements.

When selecting the best SH-WFS design in terms of the performance evaluated in this study, the choice is not immediately clear. In terms of rmsWFE the pre-AO control performance for each design is uniform. However, since the project evaluates the overall telescope design in terms of PSSN, the 5-ring design has the best performance. The PSSN varies by 0.0042 between the 2 and 5 -ring designs when including atmospheric noise. The 2-ring design has the worst performance, but the change in PSSN between the 2 and 3 -ring designs is the greatest. This makes the performance of the 3-ring design more similar to the 5-ring design than might be expected. The change in PSSN from 3 to 5-rings varies by a PSSN of only 0.0019. A 3-ring SH-WFS would greatly reduce the complexity of implementation since there are approximately 2.5 times the number of $\mathrm{SH}$ subimages in the 5-ring design at 91 lenslets per segment, while the 3-ring design has 37 lenslets per segment. There are also considerations related to detector size and wavefront spatial frequency observability for diagnostic purposes in the telescope system. This study provides another data point to the APS team when choosing the proper SH-WFS design to meet the needs of the instrument and TMT in general. While the APS team had previously selected the 5-ring design as a working baseline based on earlier analysis, a simplification to 3 lenslet rings would not sacrifice performance and would lower complexity.

\section{CONCLUSION}

The updates made to the TMT optical model incorporate WFSC correction using the telescope degrees of freedom and the interface to simulate AO correction using the NFIRAOS instrument significantly extend the capabilities of the telescope performance model. Updated performance estimates are included in this paper to show telescope calibrated and AO corrected performance of individual error terms. A simulation to vary one of the many input 
parameters in the model can quickly report the sensitivity to the variable and aid in telescope and instrument design. This process has been demonstrated by varying the APS SH-WFS design and reporting the telescope performance in terms of the metrics used by the TMT systems engineering group. A possible simplification to the SH-WFS design would not significantly impact the telescope performance in terms of these metrics.

Using the updated optical model, the next task is to simulate the telescope on and off-axis initial and maintenance alignment process. A study of the TMT alignment procedures using the telescope model will help to develop the most efficient method for aligning the telescope and evaluating the performance across telescope field of view and instrument position under a range of gravity and temperature conditions. Another future study is to use the WFSC simulation to understand the sensitivity measurement process on the real system. Atmospheric and other noise effects will limit the measurement of the as-built control degrees of freedom. These will cause non-linearities in the telescope control and a need to iterate to minimize WFE. The end-to-end telescope model will aid in determining the best methods for measuring the telescope control sensitivities and wavefront controller implementation. We can reduce the commissioning time by using the simulated Thirty Meter Telescope optical model that includes the physical effects of mirror fabrication and captures the as-built errors and sensitivities.

\section{ACKNOWLEDGMENTS}

The research was carried out in part at the Jet Propulsion Laboratory, California Institute of Technology, under a contract with the National Aeronautics and Space Administration. The TMT Project gratefully acknowledges the support of the TMT collaborating institutions. They are the Association of Canadian Universities for Research in Astronomy (ACURA), the California Institute of Technology, the University of California, the National Astronomical Observatory of Japan, the National Astronomical Observatories of China and their consortium partners, and the Department of Science and Technology of India and their supported institutes. This work was supported as well by the Gordon and Betty Moore Foundation, the Canada Foundation for Innovation, the Ontario Ministry of Research and Innovation, the National Research Council of Canada, the Natural Sciences and Engineering Research Council of Canada, the British Columbia Knowledge Development Fund, the Association of Universities for Research in Astronomy (AURA), the U.S. National Science Foundation and the National Institutes of Natural Sciences of Japan.

\section{REFERENCES}

1. D. Redding et al., "MACOS manual (Modeling and Analysis for Controlled Optical Systems)," NASA JPL D-9816, internal document $\mathbf{5}, 1999$.

2. C. Nissly, B. Seo, M. Troy, G. Angeli, J. Angione, I. Crossfield, B. Ellerbroek, L. Gilles, N. Sigrist, and L. Wang, "High-resolution optical modeling of the Thirty Meter Telescope for systematic performance trades," Proc. SPIE 7017, p. 70170U, June 2008.

3. B.-J. Seo, C. Nissly, G. Angeli, B. Ellerbroek, J. Nelson, N. Sigrist, and M. Troy, "Analysis of Normalized Point Source Sensitivity as Performance Metric for Large Telescopes," Applied Optics 48, pp. 5997-6007, 2009.

4. M. Troy, G. Chanan, S. Michaels, R. Bartos, G. Bothwell, R. Hein, M. Radin, J. Roberts, J. M. Rodgers, L. Scherr, B.-J. Seo, and D. Zimmerman, "A conceptual design for the Thirty Meter Telescope Alignment and Phasing System," Proc. SPIE, June 2008.

5. G. Chanan, M. Troy, and I. Crossfield, "Predicted measurement accuracy of the TMT Alignment and Phasing System," TMT Project Communication TMT.CTR.PRE.07.007.REL01, Feb 2007.

6. G. Chanan, "Design of the Keck Observatory alignment camera," Proc. SPIE 1036, p. 59, 1988.

7. e. a. Byoung-Joon Seo, "Proposal for TMT M2 Polishing Specification (Draft) ," TMT Project Communication TMT.SEN.SPE.12.005.DRF01, Oct 2012.

8. B. Seo, C. Nissly, G. Angeli, D. MacMynowski, N. Sigrist, M. Troy, and E. Williams, "Investigation of primary mirror segment's residual errors for the Thirty Meter Telescope," Proc. SPIE 7427, Aug. 2009. 